ANL/IPNS/CP_- 89076

$$
\text { CONF-960796--1 }
$$

\title{
A Laser Spectroscopic Study of Nd-doped Zirconia*
}

\author{
C.-K. Loong and G. K. Liu \\ Argonne National Laboratory, Argonne, Illinois 60439-4814, U. S. A. \\ M. Ozawa and S. Suzuki \\ Nagoya Institute of Technology, Tajimi, Gifu, 507, Japan
}

The submitted manuscript has been authored by a contractor of the U. S. Government under contract No. W-31-109-ENG38. Accordingly, the U. S. Government retains a nonexclusive, royalty-free license to publish or reproduce the published form of this contribution, or allow others to do so, for U. S. Government purposes.

Presented at

The 21st Rare Earth Research Conference

7-12 July 1996, Duluth, Minnesota, U. S. A.

Proceedings to be published in J. Alloys \& Compounds

*Work supported by U. S. Department of Energy, BES, contract No. W-31-109-ENG-38

Corresponding author:

Chun Loong

IPNS, Bldg. 360

Argonne National laboratory

9700 S. Cass Ave

Argonne, IL 60439-4814

U.S. A.

Tel: 708-252-5596

FAX: 708-252-4163

Email: loong@anlpns.pns.anl.gov 
Manuscript PS-22, to be presented in the 21th Rare Earth Research Conference and to be published in J. Alloys \& Compounds

\title{
A Laser Spectroscopic Study of Nd-doped Zirconia
}

\author{
C.-K. Loong and G. K. Liu \\ Argonne National Laboratory, Argonne, Illinois 60439-4814, U. S. A. \\ M. Ozawa and S. Suzuki
}

Nagoya Institute of Technology, Tajimi, Gifu, 507, Japan

\begin{abstract}
High-surface-area rare-earth (RE) modified zirconia powders prepared by solution methods can be used as catalytic support of noble metals and as electrolyte oxygen sensors in an automobile exhaust-emission-control system. A previous neutron-scattering study showed that substituting zirconium with trivalent RE ions not only stabilizes the cubic and tetragonal phases over a wide range of temperatures but also creates oxygen vacancies in the RE-Zr oxide solid solution. This work focuses on the fluorescence of $\mathrm{Nd}$ in $\mathrm{Nd}_{0.1} \mathrm{Zr}_{0.9} \mathrm{O}_{1.95}$ powders under laser excitation of the $\mathrm{Nd}^{3+}$ ground state to the ${ }^{4} \mathrm{G}_{7 / 2}$ states. Distinct features were observed at $8 \mathrm{~K}$ in the ${ }^{4} \mathrm{I}_{9 / 2} \rightarrow{ }^{4} \mathrm{G}_{7 / 2}$ excitation and ${ }^{4} \mathrm{~F}_{3 / 2} \rightarrow{ }^{4} \mathrm{I}_{9 / 2}$ emission spectra using two sets of incident and emission frequencies, respectively. The results are discussed in terms of site-sensitive local structures surrounding the $\mathrm{Nd}$ ions in the two-phased oxide structure.
\end{abstract}

Keywords: site-selective spectroscopy, fluorescence, rare-earth modified zirconia, zirconia catalytic support 


\section{Introduction}

Rare-earth dopants in zirconias play an essential role in tailoring material properties to suit different demands for technological applications. For high-surface-area zirconia used as a catalytic support in automobile exhaust-emission-control, rare-earth modification enhances structural stability and prevents sintering of the material at high temperatures. This is achieved in part by stabilizing the cubic and tetragonal phases in the $\mathrm{RE}-\mathrm{ZrO}_{2}$ system. Furthermore, since the dopant cations ( $3+\mathrm{RE}$ ions) have a lower valency than the $\mathrm{Zr}(4+)$ ions, oxygen vacancies are formed to preserve the overall electronic neutrality of the lattice. These vacancies give rise to adsorption sites and strong metal-support interactions that are needed for effective catalytic functions. Ionic conductivity resulted from oxygen diffusion via vacancy sites in response to a dynamic oxygen environment has led to the development of zirconia electrolyte oxygen sensors. Recently, Ozawa and coworkers reported the preparation of high-surface-area $\mathrm{RE}-\mathrm{ZrO}_{2}$ powders from a coprecipitation method and studied the accelerating effect of $\mathrm{CO}$ oxidation from an $\mathrm{Fe}$ catalyst supported by $\mathrm{Nd}$-doped $\mathrm{ZrO}_{2} \cdot[1-2]$

Knowledge of microscopic crystal phases and vacancy-induced defect structure in RE$\mathrm{ZrO}_{2}$ is essential to the eventual understanding of the surface chemistry and catalytic reactions in zirconia-supporting catalysts. Spectroscopic studies have provided valuable information in this regard. A recent neutron-scattering study has characterized the mixed cubic and tetragonal crystal phases, defects and dynamics of adsorbed hydroxyl groups and water in La- and Nd$\mathrm{ZrO}_{2}$. [3] While the neutron results have provided useful insights into the global structures, information regarding local structural fluctuations is not complete. Site-selective laser spectroscopy is a powerful method for identifying local structure of RE ions in solids. Laserinduced fluorescence transitions between the $4 f$ electronic states of a $\mathrm{RE}$ ion provide fingerprint energy-level patterns characteristic of the local crystalline environment surrounding the ion. Ions on different lattice or defect sites can be easily identified. Particularly, the high sensitivity of this optical technique permits the detection of RE dopants of trace-level concentration $(\sim 0.1$ 
atm\%). In the paper we report a site-selective laser spectroscopic study of the local environment of $\mathrm{RE}$ ions in the $\mathrm{Nd}-\mathrm{ZrO}_{2}$ system.

The energy level splitting of a RE ion in a solid bears a signature of the local environment surrounding the ion. As a result of vacancy-induced defects and phase inhomogenities in $\mathrm{Nd}_{0.1} \mathrm{Zr}_{0.9} \mathrm{O}_{1.95}$, trivalent $\mathrm{Nd}^{3+}$ ions occupying the $\mathrm{Zr}^{4+}$ sites exhibit different sets of crystal-field energy splitting. A superposition of optical transitions between these sets of crystal-field states gives rise to large inhomogeneous broadening of the linewidths. A narrow band laser, on the other hand, can be tuned to excite the ions that have the same energies between the ground state and the excited state. Assuming that there is a correlation between the crystalfield energy splitting and the surrounding local crystalline structure for each $\mathrm{Nd}^{3+}$ ion, this spectrally selective excitation thus permits the detection of a subset of ions having similar local

environments. The present study aims at probing the local structural properties of $\mathrm{Nd}^{3+}$ ions in $\mathrm{Nd}_{0.1} \mathrm{Zr}_{0.9} \mathrm{O}_{1.95}$ and examining the optical data in light of findings obtained from complementary spectroscopic techniques.

\section{Results and Discussion}

Nd $10-$ mol\% doped into zirconia powders, $\mathrm{Nd}_{0.1} \mathrm{Zr}_{0.9} \mathrm{O}_{1.95}$, were prepared by a coprecipitation technique described elsewhere. [1] The BET surface areas of these powders heat-treated at 600 and $800^{\circ} \mathrm{C}$ for $3 \mathrm{~h}$ in air were determined to be $73 \pm 0.8$ and $51 \pm 0.7 \mathrm{~m} / \mathrm{g}$ by nitrogen adsorption at $77 \mathrm{~K}$. A dried powder sample was sealed inside a quartz tubing which in turn was mounted in an optical cryostat for low-temperature $(8 \mathrm{~K})$ measurements. A tunable dye laser pumped by a pulsed Nd:YAG laser was used in the fluorescence experiments. The laser linewidth was $0.4 \mathrm{~cm}^{-1}$. Fluorescence emission from the lowest ${ }^{4} \mathrm{~F}_{3 / 2}$ multiplet of $\mathrm{Nd}^{3+}$ ions was dispersed by a monochromator and detected by a cooled photomultiplier. Signals from the photomultiplier were averaged using a DEC minicomputer. The optogalvanic effect in a uranium-argon hollow-cathode lamp was used for a wavelength calibration of the excitation spectra. 
First, we sought site-selective excitation of $\mathrm{Nd}^{3+}$ from the ${ }^{4} \mathrm{I}_{9 / 2}$ ground state to the ${ }^{4} \mathrm{G}_{7 / 2}$ excited state at an energy level near $19000 \mathrm{~cm}^{-1}$. The fluorescence intensities of the ${ }^{4} \mathrm{~F}_{3 / 2} \rightarrow$ ${ }^{+} I_{9 / 2}$ emission at a given wavelength was monitored as the laser incident energy was scanned over the 18500-19800 $\mathrm{cm}^{-1}$ region, and the process was repeated over a range of emission wavelengths. In a $\mathrm{Nd}_{0.1} \mathrm{Zr}_{0.9} \mathrm{O}_{1.95}$ sample heat treated at $600^{\circ} \mathrm{C}$, we obtained two dominant sets of excitation spectra at $8 \mathrm{~K}$ at corresponding emission energies of $11205 \mathrm{~cm}^{-1}$ and $11396 \mathrm{~cm}^{-1}$, as shown in Fig. 1. For a $\mathrm{Nd}^{3+}$ ion in $4 \mathrm{f}^{3}$ electronic configuration, the Kramers degeneracy cannot be lifted by crystal-field interaction. Therefore, the crystal-field levels are at least two-fold degenerate. For a single ionic site symmetry lower than cubic point-symmetry, we expect four lines in the lower energy region $\left(18400-18900 \mathrm{~cm}^{-1}\right)$ of the excitation spectrum corresponding to transitions from the ground doublet of the ${ }^{4} \mathrm{I}_{9 / 2}$ multiplet to the four doublets of the ${ }^{4} \mathrm{G}_{7 / 2}$ excited states. The lines above $18900 \mathrm{~cm}^{-1}$ correspond to the transitions to the ${ }^{4} \mathrm{G}_{9 / 2}$ and ${ }^{2} \mathrm{~K}_{13 / 2}$ multiplets. The two distinct excitation spectra (see Fig. 1) indicate that the observed excitations originate from $\mathrm{Nd}^{3+}$ ions on two distinct sites rather than from a unique environment. We denote the two site-configurations and the corresponding spectra as A and B in Fig. 1. The line with lowest energy in each of the two excitation spectra corresponds the energy of the lowest crystalfield level within the ${ }^{4} G_{7 / 2}$ multiplet. For site $A$, this level is at $18450 \mathrm{~cm}^{-1}$, and for site $B$ the level is at $18600 \mathrm{~cm}^{-1}$. Thus the energy-level difference between the two $\mathrm{Nd}^{3+}$ sites is $250 \mathrm{~cm}^{-1}$. Such differences in the crystal-field energy levels and in the splitting patterns imply rather different local environments for the two $\mathrm{Nd}^{3+}$ sites.

The two $\mathrm{Nd}^{3+}$ environments were also detected in the emission spectra. At a fixed incident laser wavelength, fluorescence was measured over a range of emission frequencies. Again two different emission spectra were obtained. The fluorescence emission from the metastable state ${ }^{4} \mathrm{~F}_{3 / 2}$ to the ${ }^{4} \mathrm{I}_{9 / 2}$ ground state is shown in Fig. 2 . The laser excitation was set at $18552 \mathrm{~cm}^{-1}$ for site A spectrum and at $18941 \mathrm{~cm}^{-1}$ for site B spectrum. In both emission spectra two lines were observed with a separation of less than $60 \mathrm{~cm}^{-1}$ which corresponds to the crystal- 
field splitting of the $\mathrm{Nd}^{3+}$ ground multiplet. The higher-energy line, which measures the energy level of the emitting states, is $11205 \mathrm{~cm}^{-1}$ for site A and 11420 for site B. Therefore, a difference of $215 \mathrm{~cm}^{-1}$ was obtained for the two $\mathrm{Nd}^{3+}$ sites in the ${ }^{4} \mathrm{~F}_{3 / 2}$ emitting states as compared with 250 $\mathrm{cm}^{-1}$ for the ${ }^{4} \mathrm{G}_{7 / 2}$ excited states. We also observed similar excitation and emission spectra from another $\mathrm{Nd}_{0.1} \mathrm{Zr}_{0.9} \mathrm{O}_{1.95}$ powder that was heat treated at $800^{\circ} \mathrm{C}$. This implies that the local structures were not altered significantly by heat treatment at such a higher temperature.

Two nominal crystal phases (cubic and tetragonal) in the Nd-doped zirconia powders were observed previously in a neutron-scattering study. [3] In the $600^{\circ} \mathrm{C}$ heat-treated $\mathrm{Nd}_{0.1} \mathrm{Zr}_{0.9} \mathrm{O}_{1.95}$ sample, molar fractions of 58.7 and $41.3 \%$ were found for the cubic and tetragonal phases, respectively. In the present experiment, we estimated that the average fluorescence intensities of site- $\mathrm{B} \mathrm{Nd} d^{3+}$ ions were about 8 times stronger than those of the site- $\mathrm{A}$ $\mathrm{Nd}^{3+}$ ions. Assuming a fluorescence intensity proportional to the number of ions in the sample, this result suggests that site- $\mathrm{B} \mathrm{Nd}^{3+}$ ions may be associated with the cubic phase. However, crystal-field selection rules for a cubic site-symmetry allows fewer lines than those observed in the site-B spectra. Therefore, the geometry of the Nd ions in the cubic phases is locally distorted to a lower symmetry than the crystallographic symmetry. In fact, local distortions around atomic sites in rare-earth modified zirconias are well-known due to charge-compensation effect in the lattice. An analysis of the diffuse-scattering background of the previous neutrondiffraction measurements using a Fourier-filtering technique provided evidence of static, oxygen vacancy-induced atomic displacements along the pseudocubic $\langle 111\rangle$ and other symmetry directions. [3]

In all cases the observed linewidths vary from $\sim 50$ to $200 \mathrm{~cm}^{-1}$, which are much larger than the homogeneous linewidths expected from a crystalline compounds. This underscores the extensive distribution of local environments over the $\mathrm{Nd}$ sites, in agreement with evidence revealed by neutron and $x$-ray diffraction experiments. [3-4] The average crystalline grain sizes for the tetragonal and cubic phases was 60 and $184 \AA$ respectively according to previous 
neutron-diffraction data. These small grain sizes imply a considerable fluctuation of local environments at the grain boundaries. It is thus not surprising to find the broad and overlapping lines in the fluorescence spectra.

In addition to the presence of two crystal phases (tetragonal and cubic) in these Nd-doped zirconia powders, small-angle neutron scattering studies revealed distinct character in the localdensity variation over two length scales. [5] Such finding is consistent with the two major poresize distributions deduced from a BET adsorption-desorption measurements. [6] Furthermore, neutron and infrared spectroscopy $[3,7]$ as well as ab initio Hartree-Fock calculations [8] showed that the surface of zirconia powders is covered by chemisorbed hydroxyl species formed in two configurations. Therefore, it is tempting to correlate the two site-selected spectra of the present study with the two regimes of local structures found in other experiments. More laser experiments on $\mathrm{Nd}-\mathrm{ZrO}_{2}$ powders with controlled $\mathrm{OH}$ and water coverage are under way to clarify the effects of surface chemistry on local structures.

\section{Acknowledgment}

We thank C. W. Williams for his assistance in preparing the sample cells for the laser experiments and J. V. Beitz for many helpful discussions. Work performed at Argonne National Laboratories is supported by the U. S. DOE, Basic Energy Sciences under Contract Nos. W-31109-ENG-38. 


\section{References}

1. M. Ozawa and M. Kimura, J. Less-Common Met. 171, 195 (1991).

2. M. Ozawa, M. Kimura, and A. Isogai, J. Alloys Compounds 193, 73 (1993); and United State Patent No. 5,075,276 (1991).

3. C.-K. Loong, J. W. Richardson, Jr., and M. Ozawa, J. Catal. 157, 634 (1995).

4. M. Ozawa, S. Susuki, and C.-K. Loong, J. Mater. Sci. Lett. 14, 796 (1995).

5. C.-K. Loong, M. Ozawa, S. Suzuki, P. Thiyagarajan, J. Richardson, Jr., and J. Nipko (in press).

6. M. Ozawa et al. (to be published).

7. See references $40-52$ cited in 3 .

8. R. Orlando, C. Pisani, C. Roetti, and E. Stefanovich, Phys. Rev. B 45, 592 (1992); and R. Orlando, C. Pisani, E. Ruiz, and P. Sauter, Surf. Sci. 275, 482 (1992). 


\title{
Figure Captions:
}

Figure 1 Laser-excitation spectra of the ${ }^{4} \mathrm{I}_{9 / 2}$ to ${ }^{4} \mathrm{G}_{7 / 2}$ transitions of $\mathrm{Nd}^{3+}$ in $\mathrm{Nd}_{0.1} \mathrm{Zr}_{0.9} \mathrm{O}_{1.95}$ at $8 \mathrm{~K}$ monitored with fluorescence emission energies of $11205 \mathrm{~cm}^{-1}$ and $11396 \mathrm{~cm}^{-1}$, respectively, for site $\mathrm{A}$ and site $\mathrm{B}$.

Figure 2 The emission spectra of the ${ }^{4} \mathrm{~F}_{3 / 2}$ to ${ }^{4} \mathrm{I}_{9 / 2}$ transitions of $\mathrm{Nd}^{3+}$ in $\mathrm{Nd}_{0.1} \mathrm{Zr}_{0.9} \mathrm{O}_{1.95}$ at incident laser energies of $18552 \mathrm{~cm}^{-1}$ for site $A$ and $18941 \mathrm{~cm}^{-1}$ for site $B$. The observed fluorescence intensity for site $B$ was divided by a factor of $\sim 8$ in order to bring it on a scale comparable to the intensity for site A.

\section{DISCLAIMER}

\begin{abstract}
This report was prepared as an account of work sponsored by an agency of the United States Government. Neither the United States Government nor any agency thereof, nor any of their employees, makes any warranty, express or implied, or assumes any legal liability or responsibility for the accuracy, completeness, or usefulness of any information, apparatus, product, or process disclosed, or represents that its use would not infringe privately owned rights. Reference herein to any specific commercial product, process, or service by trade name, trademark, manufacturer, or otherwise does not necessarily constitute or imply its endorsement, recommendation, or favoring by the United States Government or any agency thereof. The views and opinions of authors expressed herein do not necessarily state or reflect those of the United States Government or any agency thereof.
\end{abstract}




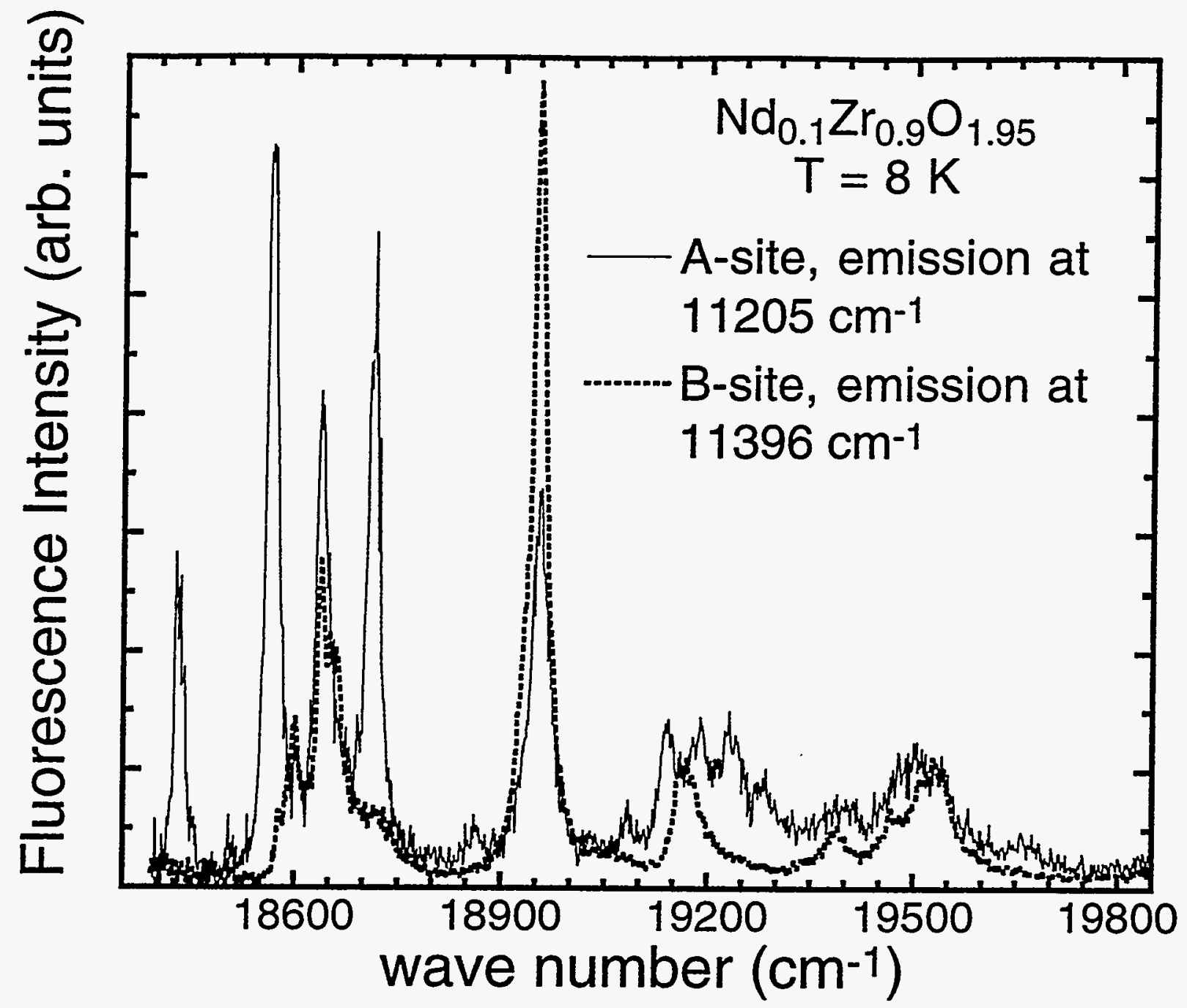

PS-22, Loong et al., "A laser spectroscopic ...", Fig. 1 


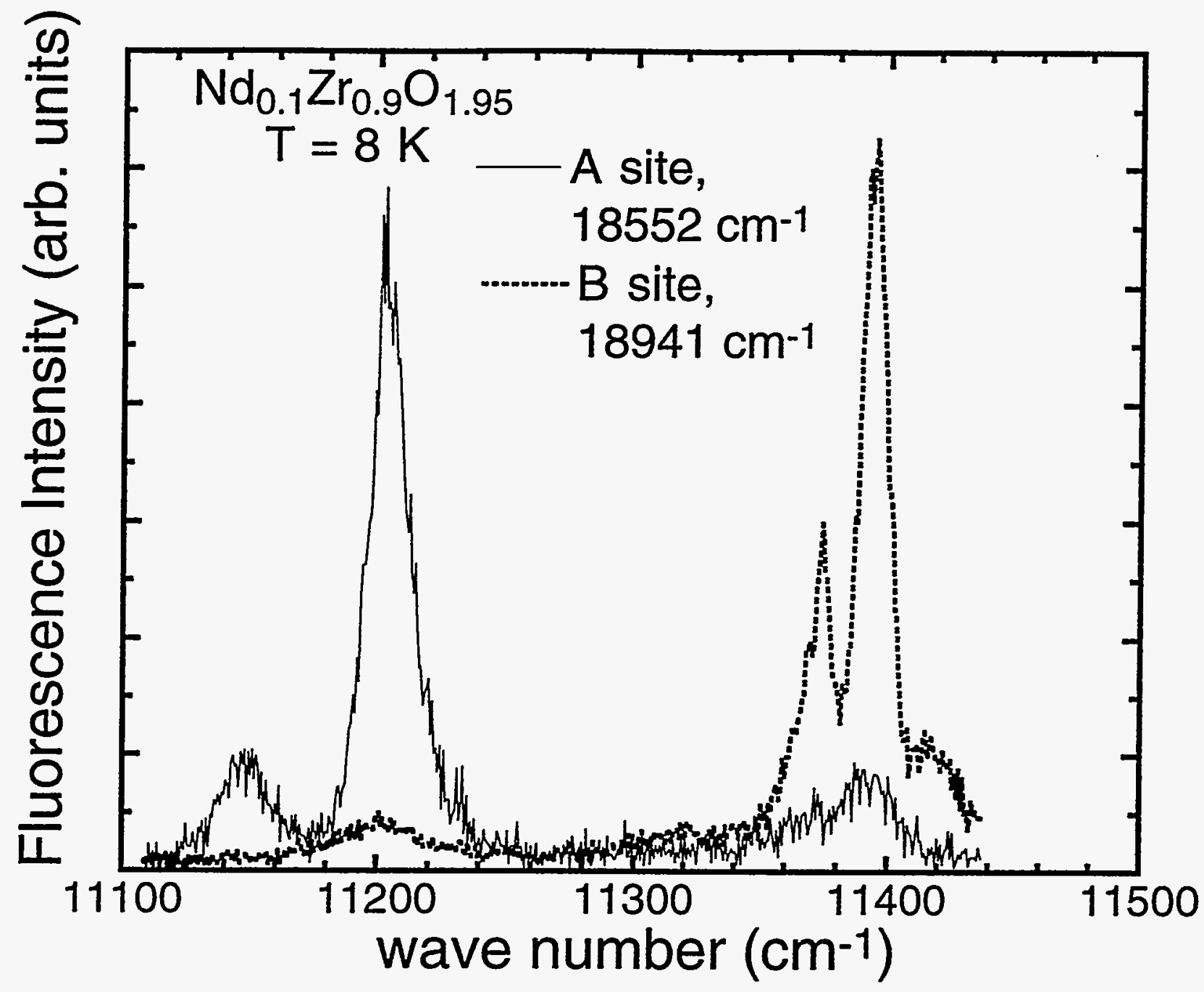

PS-22, Loong et al., "A laser spectroscopic ...", Fig. 2 